\title{
Opioid utility for dyspnea in chronic obstructive pulmonary disease: a complicated and controversial story
}

\author{
Nicholas T. Vozoris ${ }^{1,2,3,4}$ \\ ${ }^{1}$ Division of Respirology, Department of Medicine, St. Michael's Hospital, Toronto, Ontario, Canada; ${ }^{2}$ Keenan Research Centre in the Li Ka Shing \\ Knowledge Institute, St Michael's Hospital, Toronto, Ontario, Canada; ${ }^{3}$ Department of Medicine, University of Toronto, Toronto, Ontario, Canada; \\ ${ }^{4}$ ICES, Toronto, Ontario, Canada \\ Correspondence to: Dr. Nicholas T. Vozoris, MHSc, MD, FRCPC. Division of Respirology, Department of Medicine, St. Michael's Hospital, 30 Bond \\ Street, Toronto, Ontario M5B 1W8, Canada. Email: nick.vozoris@utoronto.ca.
}

\begin{abstract}
Chronic obstructive pulmonary disease (COPD) is common chronic respiratory disorder, predominantly caused by exposure to cigarette smoke or biomass fuels, and it usually affects older adults. Dyspnea in COPD that is unresponsive to traditional management is a challenging disease complication for both the patient and the health care professional. Off-label use of opioids has been advocated as a pharmacotherapy strategy for refractory dyspnea. However, negative respiratory outcomes are a potential concern with opioids drugs, especially among individuals with COPD. In this review, randomized controlled trials evaluating opioid efficacy among individuals with COPD are reviewed and critically analyzed, and data from observational drug safety studies is also presented. In summary, the evidence in support of using opioids for refractory dyspnea in COPD is minimal and weak, and there is mounting data demonstrating that opioids are associated with increased respiratory-related morbidity and mortality in this population. Therefore, current evidence does not support the broad application of opioids for refractory dyspnea among individuals with COPD. However, there may be subsets of individuals that experience modest improvement in dyspnea with opioids, and better understanding predictors and mechanisms of such opioid responsiveness should be a focus of future research endeavours.
\end{abstract}

Keywords: Chronic obstructive pulmonary disease (COPD); dyspnea; opioid

Submitted Oct 23, 2019. Accepted for publication Nov 25, 2019.

doi: 10.21037/apm.2019.11.04

View this article at: http://dx.doi.org/10.21037/apm.2019.11.04

\section{What is chronic obstructive pulmonary disease (COPD) and how are opioids potentially relevant to its management?}

COPD (also known as chronic bronchitis or emphysema) is estimated to affect upwards of $10 \%$ of individuals ages 40 years and older globally (1). Chronic airway inflammation and/or alveolar destruction are the pathologic hallmarks of COPD, resulting in airflow obstruction and lung hyperinflation, and these abnormalities can be progressive over time and are only partially reversible (2). Recurrent exposure to cigarette smoke and biomass fuels are the two most causes of COPD world-wide (2). COPD is commonly characterized by chronic respiratory symptoms (including dyspnea, cough and phlegm production), as well as recurrent acute respiratory exacerbations, during which individuals experience a heightened degree of respiratory symptoms (2). Half or more of acute respiratory exacerbations are estimated to be related to infection in the tracheobronchial tree (2). Exacerbations are associated with increased prescription medication use (2), emergency room presentations and hospital admissions (3), impairment in quality of life (4) and mortality (5). Although COPD is primarily a respiratory disorder, extra-pulmonary manifestations for various reasons also commonly occur, including but not limited to, chronic musculoskeletal pain, insomnia, and depression and anxiety (2). There is presently no cure for COPD (apart from lung transplantation), although there are 
various medications (primarily inhaled) that can help lessen troublesome respiratory symptoms, decrease chances of acute exacerbation and improve lung function (2).

Refractory dyspnea (defined as dyspnea that occurs despite optimization of traditional medical management, such as inhaled medications) is a frequently encountered and challenging problem in COPD, estimated to occur in about $50 \%$ of individuals with advanced stage disease (6). Refractory dyspnea has profound implications on patient quality of life and contributes to significant psychological distress (7). Although an off-label usage, there is a theoretical role for opioids in the management of refractory dyspnea. By binding to opioid receptors that are heavily concentrated in the medulla, where respiratory rhythm is generated, opioids may theoretically lessen refractory dyspnea by decreasing minute ventilation $(8,9)$ and/or dulling respiratory response to chemoreceptor stimulation from hypoxemia and hypercapnea (10). Opioid receptors are also located in the deeper cerebral cortex (e.g., insula, thalamus, anterior cingulate cortex, etc.) and opioid action at these sites may help modulate the perception of dyspnea $(11,12)$. Several advanced dyspnea management guidelines (13-15) and international COPD guidelines (2) have advocated using opioids for refractory dyspnea. While opioids are commonly prescribed to individuals with COPD $(16,17)$, most of this drug use is for the management of musculoskeletal pain $(16,17)$. In contrast, there is some evidence indicating that physicians hesitate to prescribe opioids for refractory dyspnea in advanced COPD (18-20), in part out of concern for potential adverse patient outcomes $(18,19)$.

Two methods of opioid prescribing for refractory dyspnea in COPD are outlined in the existing literature. The first method involves once-daily dosing of a sustainedrelease oral morphine tablet, $10-30 \mathrm{mg}$ (21-23). The second method involves standing dosing of immediaterelease liquid morphine, starting at $0.5 \mathrm{mg}$ twice daily, with possible up-titration on a weekly basis based on clinical reevaluation, followed by possible eventual substitution with a sustained-release preparation $(15,24)$. The former opioid prescription method has been more systematically evaluated (21-23) and there have been no head-to-head evaluations of the aforementioned two opioid prescribing strategies. It has been argued that use of a once-daily, sustained-release morphine preparation for refractory dyspnea is the superior prescribing strategy of the two (25), because shorter-acting opioid formulations are known to be associated with poorer steady-state blood levels and greater drug level peaks (26), thereby increasing the risk for suboptimal response and/or adverse effect.

\section{A review of the evidence on opioid efficacy for refractory dyspnea in COPD}

Three meta-analyses of randomized, double-blind, placebocontrolled trials of opioid therapy for refractory dyspnea have been published thus far: Jennings et al. in 2002 (27); Ekström et al. in 2015 (28); and, Barnes et al. in 2016 (29). Most trials included in these meta-analyses involved about 10-20 subjects each and the intervention arms in most consisted of a short-acting opioid for a few days. The earliest meta-analysis by Jennings et al. (27) reported no significant reduction in dyspnea scores with opioids versus placebo, when trials involving only COPD patients were considered [standard mean difference (SMD) in dyspnea scores between intervention versus placebo was -0.26 , with a $95 \%$ confidence interval (CI) of -0.44 to 0.08 ]. However, it should be noted that the COPD studies in this pooled analysis evaluated non-nebulized or nebulized opioids, and it has been consistently demonstrated that nebulized opioids are not effective at reducing dyspnea in COPD (27-29). In contrast, the second meta-analysis by Ekström et al. (28) (which only considered trials involving individuals with COPD and separately considered non-nebulized and nebulized opioids) reported a statistically significant improvement in dyspnea scores with non-nebulized opioids over placebo. This positive finding appears to have been largely driven by including data from the Abernethy et al. trial (21) [which was published after the earlier Jennings et al. review (27)] and the Abernethy et al. study (21) will be scrutinized in more detail further down. Although Ekström et al.'s review (28) reported a statistically significant and favourable result for non-nebulized opioids for dyspnea in COPD, it is important to highlight that the effect size observed was in fact on the small side. Specifically, the SMD was reported to be -0.34 (28), and by convention an effect size of at least 0.50 is needed to reach a level of 'medium' effect (30). Finally, similar to the earlier Jennings et al. review (27), the most recent meta-analysis by Barnes et al. (29) reported no significant reduction in dyspnea scores with opioids versus placebo, when studies involving only COPD patients were considered. However, the Barnes et al. analysis (29) has been criticised for inappropriate methodology, specifically, that data from cross-over trials had not been properly accounted for (31). All three metaanalyses concluded that opioids did not significantly 
improve objectively measured exercise capacity in advanced COPD (27-29) and this finding raises some doubt about the clinical significance of any possible small improvement in dyspnea intensity reported with opioids.

The Abernethy et al. trial (21), which is largely responsible for driving the positive result in Ekström et al.'s meta-analysis (28), deserves further examination. This was a randomized, double-blind, placebo-controlled, crossover trial evaluating sustained release oral morphine $20 \mathrm{mg}$ for four days in 42 participants with refractory dyspnea ( $88 \%$ of whom had COPD and $12 \%$ of whom had another chronic condition). The primary outcome was morning and evening sensation of dyspnea, measured on a visual analogue scale from $0-100 \mathrm{~mm}$, and secondary efficacy outcomes included self-reported sleep disturbance by dyspnea, exercise tolerance as per the modified Medical Research Council (mMRC) scale and overall sense of well-being. With respect to the primary outcome, significantly improved morning and evening dyspnea scores were observed with morphine versus placebo [morning scores were 6.6 out of $100 \mathrm{~mm}$ better (95\% CI, 1.6-11.6 mm) and evening scores were 9.5 out of $100 \mathrm{~mm}$ better (95\% CI, 3.0-16.1 mm)] (21). However, the clinical significance of these findings is questionable, as a less than 10 points improvement out of 100 on a visual analogue scale falls below what is considered to be a minimum clinically important difference (a $10-20 \mathrm{~mm}$ change is considered to be minimally clinically important) (32). With respect to secondary outcomes, while significantly fewer participants reported sleep disturbance by breathlessness when receiving opioid over placebo, mMRC score and overall sense of well-being were not significantly different (21).

Following publication of the aforementioned metaanalyses, the largest clinical trial to-date of opioids for dyspnea in COPD was completed (23). This was a multisite, randomized, double-blind, placebo-controlled, parallel-arm trial evaluating sustained release oral morphine $20 \mathrm{mg}$ for seven days among 284 subjects with chronic mMRC class 2 (or greater) dyspnea, approximately $60 \%$ of whom had COPD and the remainder other chronic conditions, with recruitment from respiratory and palliative care services. The primary outcome was breathless intensity now, measured on a visual analogue scale from 0-100 $\mathrm{mm}$, and some of the secondary efficacy outcomes included worst breathlessness intensity in the past $24 \mathrm{~h}$, average breathlessness intensity in the past $24 \mathrm{~h}$, participant overall impression of functional status and quality of life via questionnaire. There was no significant difference in the primary outcome between the morphine and placebo groups (SMD $-0.15 ; 95 \%$ CI, -4.59 to 4.29 ), nor in any of the secondary efficacy outcomes (23). In a subgroup analysis involving those individuals with $\mathrm{mMRC}$ class 3 dyspnea and greater, no significant differences were again found for the primary and secondary outcomes between the morphine and placebo groups (23).

To whom any possible small benefits of opioid therapy apply is a separate legitimate question raised by the efficacy studies published to-date. Individuals with a history of acute respiratory exacerbation, hypercapnea, comorbid cardiovascular or neuropsychiatric disease, and recipients of other psychoactive medications were subgroups of individuals often not included in aforementioned clinical trials of opioids in COPD. Yet, all these characteristics feature prominently among individuals with COPD (33-35), thereby raising questions about the external generalizability of any possible small benefits with opioids.

\section{A review of the evidence on respiratory-related harms associated with opioid use in COPD}

A variety of psychomotor and gastrointestinal side-effects are known to potentially occur with opioid use, including dizziness, delirium, somnolence, falls and fractures, constipation, and nausea and vomiting, especially among older adults (36-38). However, possible respiratoryrelated harm is topic of particular concern with opioid drug use among individuals with COPD. There are several potential ways that opioids may lead to respiratory harm among individuals with COPD: depression of central respiratory drive $(8,9)$; activation of peripherally-located opioid receptors in the tracheobronchial tree causing cough suppression and subsequent mucous impaction (39); possible aspiration as a result of sedation; and, negative immunomodulatory effects $(40,41)$. Potential negative opioid-related respiratory effects become even more relevant among individuals with COPD, given their baseline respiratory system compromise and their predisposition to recurrent acute respiratory exacerbations.

Although randomized controlled trials evaluating opioids in COPD have reported no major drug-related adverse respiratory events $(23,27-29)$, these studies were specifically designed to evaluate for possible opioid efficacy and they were not structured to adequately examine for possible drug harms. Features of opioid efficacy trials that limit their ability to comprehensively evaluate for possible drug harms include: relatively small numbers of study participants were 
often included (in contrast, large participant numbers are ideally needed to properly evaluate for possible drug harms, since adverse events are likely to occur in a minority of individuals); individuals at heightened risk for experiencing adverse respiratory events were often excluded, such as exacerbators, hypoventilators, those with comorbid illnesses, recipients of other types of psychoactive drugs and individuals with previous adverse reaction to opioids; there was participant drop-out during the studies (which is often due to the experiencing of adverse events) and data from these individuals were not considered in final analyses; and, low or single-dose opioid drug regimens were examined, which may not reflect how these drugs are in fact used in the 'real world'. Serious ethical considerations would also preclude use of a randomized trial design to evaluate for possible drug harms as the primary outcome. Instead, one must turn to observational research studies to gain an appreciation of possible opioid-related harms. For the purposes of drug safety assessment, observational studies overcome many of the limitations associated with randomized controlled therapy efficacy trials: they typically include larger sample sizes; they incorporate data on individuals from the broader population (including from sicker and more vulnerable individuals, who would be at greater risk for experiencing drug side-effects); there is minimal-to-no loss of participant follow-up; and, they evaluate drug utilization as it occurs in the 'real word' and not under artificial, controlled conditions (which may help protect from the occurrence of adverse events). While causation can never be firmly concluded as the explanation for any observed associations in an observational study, observational research nonetheless provides the best evidence about possible drug harms (42) and various techniques (such as covariate matching and stratification) can be used to minimize possible confounding.

One of the earliest opioid safety studies among individuals with COPD was by Ekström et al. (43) in 2014. This was a prospective cohort study, involving over 2000 Swedes with oxygen-dependent COPD, evaluating allcause hospitalization and all-cause mortality in relation to prevalent prescription opioid drug use. After adjusting for wide range COPD severity markers, there was a significantly increased risk of all-cause mortality [hazard ratio (HR) 1.21; 95\% CI, 1.02-1.44] among individuals receiving $>30 \mathrm{mg}$ oral morphine equivalents per day versus non-opioid users (43). No association was observed between all-cause mortality and receipt of $\leq 30 \mathrm{mg}$ oral morphine equivalents per day, nor was all-cause hospitalization associated with opioid use, regardless of daily dosage (43).

A subsequent retrospective cohort study of $\sim 130,000$ non-palliative older adults with COPD living in Ontario, Canada, examined the association between incident opioid prescription drug use and 30-day respiratoryrelated morbidity and mortality (44). From a drug safety perspective, incident use is a more important drug utilization pattern to examine than prevalent use. Prevalent use is more likely to be influenced by 'healthy user bias', and therefore, potentially under-estimate drug harms (i.e., drug users that suffer an adverse event and subsequently stop the drug, or die, are less likely to be prevalent users, and this bias is minimized when examining new drug users). After balancing on an extensive range of covariates between opioid recipients and non-recipients (including multiple markers of COPD severity), incident opioid use was found to be associated with significantly increased emergency room visits for COPD or pneumonia (HR 1.14; 95\% CI, 1.00-1.29), COPD or pneumonia-related mortality (HR 2.16; 95\% CI, 1.61-2.88) and all-cause mortality (HR 1.76; 95\% CI, 1.57-1.98) (44). Increased respiratory-related morbidity and mortality in association with opioid use persisted after excluding individuals with malignancy from the analysis (who would be greater risk for having poorer outcomes) and when limiting the analysis to non-exacerbators (which reflects a healthier subgroup of individuals) (44), and these results further support the credibility of the overall findings. Elevated risk of adverse respiratory outcomes was observed to occur regardless of opioid half-life duration and estimated daily dose (44). Increased rates of negative respiratory outcomes were also observed regardless of whether an opioid used was combined in a single pill with a non-opioid analgesic (like acetaminophen or aspirin), although adverse event risk was particularly heightened with use of the generally more potent opioid-only formulations (44).

A recently published case-control study of $\sim 10,000$ older Americans with COPD (45) evaluated COPDrelated hospitalization in relation to prevalent prescription opioid use in the preceding 30 days. After matching cases to controls on wide range factors, including indicators of COPD severity, opioid receipt was associated with significantly increased risk of COPD-related hospitalization (HR 1.73; 95\% CI, 1.52-1.97) (45). Hospitalization risk was magnified if a benzodiazepine had been concurrently used with an opioid (HR 2.32; 95\% CI, 1.94-2.77) (45).

Although not undertaken specifically among individuals with COPD, two final observational studies are worthy of 
mention, since they evaluated opioid drug use in relation to pneumonia risk, and since respiratory tract infection is a common cause for acute exacerbations in COPD. Among 3,000 community-dwelling older adults in the United States, Dublin et al. (46) examined the association between validated cases of pneumonia and prevalent prescription opioid drug use, using a case-control study design and adjusting for relevant potential confounders. Prevalent opioid drug use within the preceding 60 days was found to be associated with significantly greater pneumonia risk [odds ratio (OR) 1.38; 95\% CI, 1.08-1.76], particularly among those using long-acting formulations (OR 3.43; $95 \%$ CI, 1.44-8.21) or opioids with known immunosuppressive properties (e.g., codeine, morphine and fentanyl) (OR 1.88, 95\% CI, 1.26-2.79) (46). More recently, Wiese et al. (47) evaluated the association between microbiologicallyconfirmed cases of pneumococcal disease and prevalent prescription opioid drug use among 1,200 Americans, using a nested case-control study design and adjusting for relevant potential confounders. Current opioid recipients were found to have significantly increased risk of pneumococcal infection (OR 1.62; 95\% CI, 1.36-1.92), particularly incident users (OR 2.44; 95\% CI, 1.49-4.00) (47). While elevated risk of pneumococcal infection was observed across all categories of opioid duration of action, potency and daily dose evaluated (compared to non-opioid use), high potency formulations and the highest daily dose category were demonstrated to be associated with the greatest pneumococcal infection risk (47) and these findings strengthen that exposure and outcome are indeed linked.

\section{Opioid utility for dyspnea in COPD: a synthesis of the evidence on benefits and harms}

Although there is a theoretical basis for benefit (8-12) and multiple clinical practice guidelines advocate using opioids for refractory dyspnea in COPD (2,13-15), the evidence in support of opioids being effective for this purpose is in fact minimal and weak $(21,23,27-29)$. Nevertheless, one cannot exclude that opioids might offer some small benefits to certain individuals with COPD struggling with intolerable and difficult-to-manage dyspnea. Considerable evidence has also accumulated supporting that opioid use is associated with increased risk for serious adverse respiratory outcomes (among other types of side-effects) in COPD (43-47). While results of opioid drug safety research need to be taken into consideration in opioid prescribing decision- making, at the same time, one must also not go too far and take the position that opioids should never be prescribed for any reason among individuals with COPD because harm will always ensue. Perhaps the 2016 version of the Global Initiative for Chronic Obstructive Lung Disease (GOLD) guidelines (48) came close to striking an reasonable balance between opioid-related benefits and harms, acknowledging that opioids have dyspnea-relieving potential in COPD, but at that same time warning that: "morphine used to control dyspnea may have serious adverse effects and its benefits may be limited to a few sensitive subjects" (48). Unfortunately, more recent versions of the GOLD guidelines (2) depart from this balanced position and instead advocate for "wider use of palliative therapies [including opioids] that in the past have often been restricted to endof-life situations" (2). The GOLD guidelines' revised position on opioids in COPD is non-evidence-based and imprudent, as the evidence in support of opioid efficacy for dyspnea in COPD is weak, and since there is mounting data of opioid-related respiratory harms, which will likely become only more commonplace with "wider" usage.

Future research relating to opioids in COPD should focus on attempting to elucidate which factors potentially predict favourable response to opioid therapy and the neurophysiological explanations for this (and conversely, which factors predispose to opioid-related harms and why). The answers to these questions unlikely lie with particular opioid formulations being used or not used, as the current body of drug safety literature shows that adverse respiratory outcomes can occur with opioids, regardless of duration of action, potency and daily dosage. Instead, particular patient characteristics may be the more important factor influencing response to opioids and there is some early research in support of this concept. Johnson et al. (49) pooled data from four clinical trials of opioid therapy for chronic refractory breathlessness (largely, but not exclusively, involving individuals with COPD) and identified that younger age and greater breathlessness intensity were factors that predicted favourable opioid response. Intriguing research by Abdallah et al. (50) has suggested that individuals with higher levels of negative affect (i.e., depression, anxiety) may less likely favourably respond to opioid therapy and that such individuals may do better with pulmonary rehabilitation. Additional research identifying predictors of opioid response/non-response may be key to bringing clarity to the controversial and complex topic of opioid utility in COPD. 


\section{Acknowledgments}

Funding: None.

\section{Footnote}

Provenance and Peer Review: This article was commissioned by the Guest Editor (Mellar P. Davis) for the series "Opioid Utility the Other Half of Equianalgesia" published in Annals of Palliative Medicine. The article was sent for external peer review organized by the Guest Editor and the editorial office.

Conflicts of Interest: The series "Opioid Utility the Other Half of Equianalgesia" was commissioned by the editorial office without any funding or sponsorship. The author has no other conflicts of interest to declare.

Ethical Statement: The author is accountable for all aspects of the work in ensuring that questions related to the accuracy or integrity of any part of the work are appropriately investigated and resolved.

Open Access Statement: This is an Open Access article distributed in accordance with the Creative Commons Attribution-NonCommercial-NoDerivs 4.0 International License (CC BY-NC-ND 4.0), which permits the noncommercial replication and distribution of the article with the strict proviso that no changes or edits are made and the original work is properly cited (including links to both the formal publication through the relevant DOI and the license). See: https://creativecommons.org/licenses/by-nc-nd/4.0/.

\section{References}

1. Buist AS, McBurnie MA, Vollmer WM, et al. BOLD Collaborative Research Group. International variation in the prevalence of COPD (the BOLD Study): a populationbased prevalence study. Lancet 2007;370:741-50.

2. Global Initiative for Chronic Obstructive Lung Disease (GOLD). Global Strategy for the Diagnosis, Management, and Prevention of Chronic Obstructive Pulmonary Disease. 2019 Report. (Accessed October 2019). Available online: https://goldcopd.org/wp-content/ uploads/2018/11/GOLD-2019-v1.7-FINAL-14Nov2018WMS.pdf

3. Burrows B, Earle RH. Course and prognosis of chronic obstructive lung disease. A prospective study of 200 patients. N Engl J Med 1969;280:397-404.

4. Spencer S, Calverley PM, Burge PS, et al. Impact of preventing exacerbations on deterioration of health status in COPD. Eur Respir J 2004;23:698-702.

5. Connors AF Jr, Dawson NV, Thomas C, et al. Outcomes following acute exacerbation of severe chronic obstructive lung disease. Am J Respir Crit Care Med 1996;154:959-67.

6. Elkington $\mathrm{H}$, White $\mathrm{P}$, Addington-Hall J, et al. The healthcare needs of chronic obstructive pulmonary disease patients in the last year of life. Palliat Med 2005;19:485-91.

7. Elkington $\mathrm{H}$, White $\mathrm{P}$, Addington-Hall J, et al. The last year of life of COPD: a qualitative study of symptoms and services. Respir Med 2004;98:439-45.

8. Montandon G, Qin W, Liu H, et al. PreBotzinger complex neurokinin-1 receptor-expressing neurons mediate opioid-induced respiratory depression. J Neurosci 2011;31:1292-301.

9. Lalley PM. Opioid receptor agonist effects on medullary respiratory neurons in the cat: evidence for involvement in certain types of ventilatory disturbances. Am J Physiol Regul Integr Comp Physiol 2003;285:R1287-304.

10. Weil JV, McCullough RE, Kline JS, et al. Diminished ventilatory response to hypoxia and hypercapnia after morphine in normal man. N Engl J Med 1975;292:1103-6.

11. Pattinson KT. Opioids and the control of respiration. Br J Anaesth 2008;100:747-58.

12. Pattinson KT, Governo RJ, MacIntosh BJ, et al. Opioids depress cortical centers responsible for the volitional control of respiration. J Neurosci 2009;29:8177-86.

13. Lanken PN, Terry PB, Delisser HM, et al. ATS Endof-Life Care Task Force. An official American Thoracic Society clinical policy statement: palliative care for patients with respiratory diseases and critical illnesses. Am J Respir Crit Care Med 2008;177:912-27.

14. Mahler DA, Selecky PA, Harrod CG, et al. American College of Chest Physicians consensus statement on the management of dyspnea in patients with advanced lung or heart disease. Chest 2010;137:674-91.

15. Marciniuk DD, Goodridge D, Hernandez P, et al. Managing dyspnea in patients with advanced chronic obstructive pulmonary disease: a Canadian Thoracic Society clinical practice guideline. Can Respir J 2011;18:69-78.

16. Vozoris NT, Wang X, Fischer HD, et al. Incident opioid drug use among older adults with chronic obstructive pulmonary disease: a population-based cohort study. Br J Clin Pharmacol 2016;81:161-70. 
17. Ahmadi Z, Bernelid E, Currow DC, et al. Prescription of opioids for breathlessness in end-stage COPD: a national population-based study. Int J Chron Obstruct Pulmon Dis 2016;11:2651-7.

18. Rocker G, Young J, Donahue M, et al. Perspectives of patients, family caregivers and physicians about the use of opioids for refractory dyspnea in advanced chronic obstructive pulmonary disease. CMAJ 2012;184:E497-504.

19. Janssen DJ, de Hosson SM, Bij de Vaate E, et al. Attitudes toward opioids for refractory dyspnea in COPD among Dutch chest physicians. Chron Respir Dis 2015;12:85-92.

20. Ahmadi Z, Sandberg J, Shannon-Honson A, et al. Is Chronic Breathlessness Less Recognized and Treated Compared with Chronic Pain? A Case-Based Randomised Controlled Trial. Eur Respir J 2018. doi: 10.1183/13993003.00887-2018.

21. Abernethy AP, Currow DC, Frith P, et al. Randomised, double blind, placebo controlled crossover trial of sustained release morphine for the management of refractory dyspnoea. BMJ 2003;327:523-8.

22. Currow DC, McDonald C, Oaten S, et al. Once-daily opioids for chronic dyspnea: a dose increment and pharmacovigilance study. J Pain Symptom Manage 2011;42:388-99.

23. Currow D, Louw S, McCloud P, et al. Regular, sustainedrelease morphine for chronic breathlessness: a multicentre, double-blind, randomised, placebo-controlled trial. Thorax 2020;75:50-6.

24. Rocker GM, Simpson AC, Young J, et al. Opioid therapy for refractory dyspnea in patients with advanced chronic obstructive pulmonary disease: patients' experiences and outcomes. CMAJ Open 2013;1:E27-36.

25. Currow DC, Ekström M, Johnson MJ. Overdosing on immediate-release morphine solution has predictable adverse effects. Eur Respir J 2017. doi: 10.1183/13993003.01091-2017.

26. Argoff CE, Silvershein DI. A comparison of longand short-acting opioids for the treatment of chronic noncancer pain: tailoring therapy to meet patient needs. Mayo Clin Proc 2009;84:602-12.

27. Jennings AL, Davies AN, Higgins JP, et al. A systematic review of the use of opioids in the management of dyspnoea. Thorax 2002;57:939-44.

28. Ekström M, Nilsson F, Abernethy AA, et al. Effects of opioids on breathlessness and exercise capacity in chronic obstructive pulmonary disease. A systematic review. Ann Am Thorac Soc 2015;12:1079-92.

29. Barnes H, McDonald J, Smallwood N, et al. Opioids for the palliation of refractory breathlessness in adults with advanced disease and terminal illness. Cochrane Database Syst Rev 2016;3:CD011008.

30. Cohen J. Statistical Power Analysis for the Behavioral Sciences. New York: Academic Press, 1969.

31. Ekström M, Bajwah S, Bland JM, et al. One evidence base; three stories: do opioids relieve chronic breathlessness? Thorax 2018;73:88-90

32. Ries AL. Minimally clinically important difference for the UCSD shortness of breath questionnaire, Borg scale, and visual analog scale. COPD 2005;2:105-10.

33. Seemungal TA, Donaldson GC, Bhowmik A, et al. Time course and recovery of exacerbations in patients with chronic obstructive pulmonary disease. Am J Respir Crit Care Med 2000;161:1608-13.

34. Huiart L, Ernst P, Suissa S. Cardiovascular morbidity and mortality in COPD. Chest 2005;128:2640-6.

35. Vozoris NT, Fischer HD, Wang X, et al. Benzodiazepine use among older adults with chronic obstructive pulmonary disease: a population-based cohort study. Drugs Aging 2013;30:183-92.

36. Buckeridge D, Huang A, Hanley J, et al. Risk of injury associated with opioid use in older adults. J Am Geriatr Soc 2010;58:1664-70.

37. Clegg A, Young JB. Which medications to avoid in people at risk of delirium: a systematic review. Age Ageing 2011;40:23-9.

38. Papaleontiou M, Henderson CR Jr, Turner BJ, et al. Outcomes associated with opioid use in the treatment of chronic noncancer pain in older adults: a systematic review and meta-analysis. J Am Geriatr Soc 2010;58:1353-69.

39. Adcock JJ, Schneider C, Smith TW. Effects of codeine, morphine and a novel opioid pentapeptide BW443C, on cough, nociception and ventilation in the unanaesthetized guinea-pig. Br J Pharmacol 1988;93:93-100.

40. Vallejo R, de Leon-Casasola O, Benyamin R. Opioid therapy and immunosuppression: a review. Am J Ther 2004;11:354-65.

41. Wang J, Barke RA, Charboneau R, et al. Morphine impairs host innate immune response and increases susceptibility to Streptococcus pneumoniae lung infection. J Immunol 2005;174:426-34.

42. Vandenbroucke JP, Psaty BM. Benefits and risks of drug treatments: how to combine the best evidence on benefits with the best data about adverse effects. JAMA 2008;300:2417-9.

43. Ekström MP, Bornefalk-Hermansson A, Currow DC. Safety of benzodiazepines and opioids in very severe 
respiratory disease: national prospective study. BMJ 2014;348:g445.

44. Vozoris NT, Wang X, Fischer HD, et al. Incident opioid drug use and adverse respiratory outcomes among older adults with COPD. Eur Respir J 2016;48:683-93.

45. Baillargeon J, Singh G, Kuo YF, et al. Association of Opioid and Benzodiazepine Usewith Adverse Respiratory Events in Older Adults with Chronic Obstructive Pulmonary Disease. Ann Am Thorac Soc 2019; 16:1245-51.

46. Dublin S, Walker RL, Jackson ML, et al. Use of opioids or benzodiazepines and risk of pneumonia in older adults: a population-based case-control study. J Am Geriatr Soc 2011;59:1899-907.

47. Wiese AD, Griffin MR, Schaffner W, et al. Opioid

Cite this article as: Vozoris NT. Opioid utility for dyspnea in chronic obstructive pulmonary disease: a complicated and controversial story. Ann Palliat Med 2020;9(2):571-578. doi: 10.21037/apm.2019.11.04
Analgesic Use and Risk for Invasive Pneumococcal Diseases: A Nested Case-Control Study. Ann Intern Med 2018;168:396-404.

48. Global Initiative for Chronic Obstructive Lung Disease (GOLD). Global Strategy for the Diagnosis, Management, and Prevention of Chronic Obstructive Pulmonary Disease. 2016.

49. Johnson MJ, Bland JM, Oxberry SG, et al. Opioids for chronic refractory breathlessness: patient predictors of beneficial response. Eur Respir J 2013;42:758-66.

50. Abdallah SJ, Faull OK, Wanigasekera V, et al. Opioids for breathlessness: psychological and neural factors influencing response variability. Eur Respir J 2019. doi: 10.1183/13993003.00275-2019. 